\title{
Internet das Coisas Robóticas: Desenvolvimento de um protótipo para o ensino de programação
}

\author{
Gabriel Jaime Alves \\ Universidade Federal do Paraná \\ Jandaia do Sul - PR - Brasil \\ ga.jalves@ufpr.br
}

\author{
André Pinto Moreira \\ Universidade Federal do Paraná \\ Jandaia do Sul - PR - Brasil \\ moreira.andre.p@ufpr.br
}

\author{
Carlos Roberto Beleti Junior \\ Universidade Federal do Paraná \\ Jandaia do Sul - PR - Brasil \\ carlosbeleti@ufpr.br
}

\author{
Daniela Eloise Flôr \\ Instituto Federal do Paraná \\ Paranavaí - PR - Brasil \\ daniela.flor@ifpr.edu.br
}

\author{
Linnyer Beatrys Ruiz Aylon \\ Universidade Estadual de Maringá \\ Maringá - PR - Brasil \\ lbruiz@uem.br
}

\begin{abstract}
Aiming at addressing the principles of programming logic and developing problem analysis and problem solving skills, the discipline of algorithms and programming is present in the curricula of various courses, from computer science courses to engineering. In the literature, some studies have shown that such disciplines usually present high percentages of dropout and disapproval due to several factors, but mainly difficulties of abstraction as to the logic required to solve problems using programming. To contribute to these factors, it is envisaged to provide ways of teaching that help the student to reason and find solutions to problems with programming. One method that may be most effective is block programming, because it aims to facilitate the introduction of programming concepts to students. Block programming, unlike traditional programming, is more interactive and visual, consisting of blocks that represent computational instructions, where blocks are connected to others to be able to perform programming. Thus, this research presents the development of an IoRT-based prototype along with block programming to promote computational thinking, more specifically in the teaching of algorithms and programming. The internet of robotic things emerges as an emerging vision that brings together objects focused on the production of action, autonomous behavior and especially interaction. IoRT presents itself as a great ally for teaching, focusing on promoting student engagement and expanding teaching possibilities.
\end{abstract}

\section{KEYWORDS}

Internet das Coisas, Internet das Coisas Robóticas, Educação.

\section{Introdução}

Atualmente temos vivido em uma era informatizada em que as crianças já nascem inseridas no mundo digital, sendo denominadas nativas digitais. Para Mitchel Resnick (2009) usar as tecnologias com naturalidade não é o bastante para se adquirir habilidades, ou se tornar "fluentes" com as novas tecnologias, para ele é necessário que se saiba projetar, criar e se expressar por meio dessas tecnologias. Nesse sentido, a aprendizagem de programação, além de desenvolver o pensamento computacional, trabalha habilidades e competências ligadas ao raciocínio lógico e matemático, que são úteis na aprendizagem de conceitos em diversas áreas. Para Ferradin e Stephani (2012) quando se fala do ensino de programação devemos sempre buscar formas de ensino que auxiliem o aluno a encontrar seu estilo de raciocinar e encontrar soluções para problemas. Além disso, para esses autores a resolução de problemas e a construção dos algoritmos é a maior dificuldade no ensino de programação, pois exige que o aluno apresente domínio de raciocínio lógico, matemático, simbólico, e também, grande capacidade de abstração. Robins et al. (2003) observa que grande parte do esforço dedicado ao ensino de programação é focado no ensino da sintaxe e das estruturas da linguagem de programação, entretanto os autores argumentam que mais cruciais que esse conhecimento, são as estratégias para aplicá-lo. Sendo assim, este trabalho buscou desenvolver um protótipo que tem como foco auxiliar no processo de ensino-aprendizagem de conceitos computacionais, como algoritmos e programação. O protótipo foi desenvolvido tomando por base o paradigma da Internet das Coisas e Internet das Coisas Robóticas. 


\section{Internet das Coisas}

Pra I. Lee e K. Lee (2015), a Internet das Coisas (do inglês Internet of Things - IoT) é vista como uma rede global de máquinas e dispositivos capazes de interagir uns com os outros. A internet das Coisas surge como um novo paradigma que apresenta aspectos e tecnologias provenientes de diferentes abordagens. O objeto inteligente é o bloco de construção da visão da IoT. Colocando inteligência nos objetos do dia a dia, eles se transformam, e são capazes de não apenas coletar informações do ambiente, e interagir com o mundo físico, mas também de se interconectar, por meio da Internet, para trocar dados e informações (BORGIA, 2014).

\subsection{Internet das Coisas Robóticas}

Atreladas a Internet da Coisas, novas nomenclaturas surgem, visando denominar áreas específicas, assim como a Internet das Coisas Robóticas. Essa, pode ser considerada como uma infraestrutura global para a sociedade da informação, também permitindo a interconexão de serviços robóticos e as coisas robóticas com base em tecnologias existentes e em desenvolvimento (RAY, 2016). Afanasyev et al. (2019) afirmam que a Internet das Coisas Robóticas é um nível mais avançado da Internet das Coisas. Para os autores ela permite integrar tecnologias modernas de comunicação, detecção, atuação, monitoramento, processamento de dados, além de autonomia de decisão e manipulação.

\section{Desenvolvimento}

O desenvolvimento do protótipo teve como tecnologias empregadas a biblioteca Blockly, uma placa Arduino Uno, dois motores DC, uma Ponte H L298N, e um módulo bluetooth HM10 com tecnologia Bluetooth Low Energy (BLE). A Figura 1 exibe o protótipo. Todas as peças foram montadas sobre um chassi de acrílico.

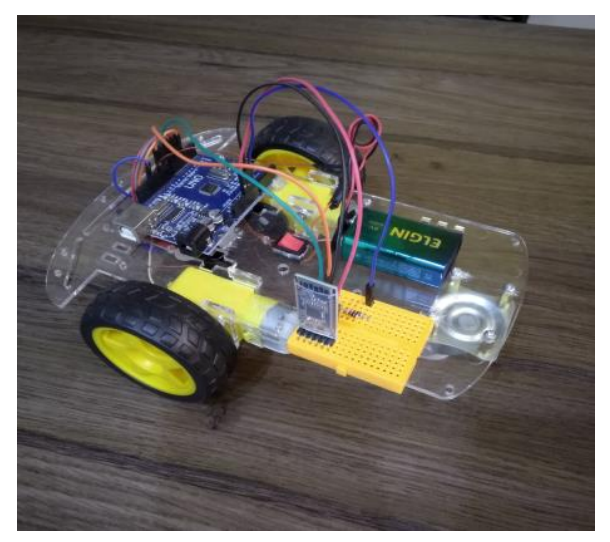

Figura 1: O Protótipo do robô.
Como descrito anteriormente, foi utilizado o Blockly ${ }^{1}$, que é um conjunto de bibliotecas que possibilita a criação de um editor visual para programação baseado em blocos. É um projeto do Google, e é um software gratuito e de código aberto, escrito em Javascript e destina-se a ser executado dentro de um ambiente de navegador web. A biblioteca permite criar blocos e adicioná-los ao ambiente de programação. Tais blocos criados podem conter novas capacidades e instruções para a construção de programas. A Block Factory ${ }^{2}$ é a página responsável por fornecer as ferramentas necessárias para a criação de um novo bloco, tanto os seus aspectos, como seu comportamento. Neste trabalho utilizamos essa página para desenvolver, a princípio, três blocos conforme listados na Figura 2, junto a interface de programação do robô. Nessa interface, por meio de um smartphone, pode-se inserir blocos e fazer a execução, e assim, quase que em tempo real o robô executa os comandos.

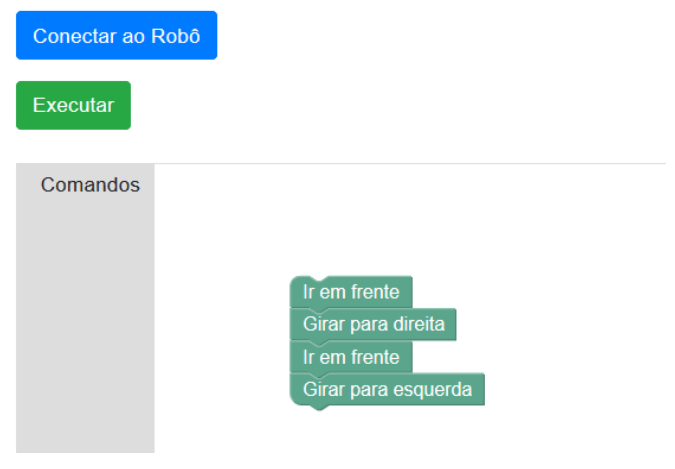

Figura 2: Interface de programação e blocos desenvolvidos.

\section{Considerações Finais}

Com o desenvolvimento do protótipo e o ambiente de programação finalizados, os próximos passos consistem em aplicar atividades voltadas para o ensino de programação com alunos de escolas públicas. O objetivo é apresentar e iniciar jovens às áreas de computação, mas especificamente Internet das Coisas Robóticas, entre outros conceitos computacionais de uma maneira mais lúdica e atrativa.

\section{REFERÊNCIAS}

[1] AFANASYEV, llya et al. Towards the internet of robotic things: Analysis, architecture, components and challenges. arXiv preprint arXiv:1907.03817, 2019.

\footnotetext{
1 https://developers.google.com/blockly

2 https://blockly-demo.appspot.com/static/demos/blockfactory
} 
[2] BORGIA, Eleonora. The Internet of Things vision: Key features, applications and open issues. Computer Communications, v. 54, p. 1-31, 2014.

[3] FERRANDIN, Mauri; STEPHANI, Simone Lilian. Ferramenta para o ensino de programação via Internet. Anais SULCOMP, v. 1, 2012.

[4] LEE, In; LEE, Kyoochun. The Internet of Things (IoT): Applications, investments, and challenges for enterprises. Business Horizons, $v$. 58, n. 4, p. 431-440, 2015.

[5] RAY, Partha Pratim. Internet of robotic things: Concept, technologies, and challenges. IEEE Access, v. 4, p. 9489-9500, 2016.

[6] RESNICK, Mitchel et al. Scratch: Programming for all. Commun. Acm, v. 52, n. 11, p. 60-67, 2009.

[7] ROBINS, Anthony; ROUNTREE, Janet; ROUNTREE, Nathan. Learning and teaching programming: A review and discussion. Computer science education, v. 13, n. 2, p. 137-172, 2003.

[8] PRENSKY, Marc. Digital natives, digital immigrants part 1. On the horizon, v. 9, n. 5, p. 1-6, 2001. 